\title{
Posicionamiento de la investigación en Ciencias Sociales
}

\author{
María Cruz Sánchez-Gómez1 ${ }^{1}$, María Victoria Martín-Cilleros ${ }^{1}$, António Pedro Costa ${ }^{2}$, \\ Francisco José García Peñalvo ${ }^{1}$. \\ mcsago@usal.es, viquimc@usal.es,pcosta@ludomedia.pt, fgarcia@usal.es. \\ ${ }^{1}$ Universidad de Salamanca, Paseo de Canalejas, 169, 37008 Salamanca. España. \\ ${ }^{2}$ Ludomedia/webQDA e CIDTFF/UA - Centro de Investigação Didática e Tecnologia na Formação de \\ Formadores, DEP/UA - Departamento de Educação e Psicologia, Universidade de Aveiro, Aveiro, Portugal.
}

DOI: 10.17013/risti.28.102-113

\begin{abstract}
Resumen: Con el objetivo de conocer la evolución de los diferentes métodos de investigación en el área de las Ciencia Sociales, se realiza un análisis de la producción científica, utilizando para ello dos bases de datos internacionales, web of science y scopus. Se ha seleccionado un periodo de los últimos veinticinco años analizando los progresos tanto en la literatura científica general como en las ciencias sociales específicamente. Se observa una creciente evolución de los métodos cualitativos y mixtos así como un ligero descenso de la productividad en general en el último año.
\end{abstract}

Palabras-clave: bibliometría; qualitativa; cuantitativa; mixto; investigación.

\section{Positioning of research in the area of Social Sciences.}

\begin{abstract}
In order to know the evolution of the different research methods in the area of Social Science, an analysis of scientific production is carried out, using two international databases, web of science and scopus. A period of the last twenty-five years is selected by analyzing progress in both the general scientific literature and the social sciences specifically. There has been a growing evolution of qualitative and mixed methods as well as a slight decrease in overall productivity over the last year.
\end{abstract}

Keywords: bibliometrics, qualitative; quantitative; mixed; research.

\section{Introducción}

La metodología en investigación científica hace referencia al modo en el cual el investigador enfoca el problema y busca las respuestas. Implica la reflexión sobre cómo obtener conocimiento, qué se debe hacer y cómo realizarlo. Los propósitos, supuestos e intereses han determinado la metodología a seguir en una investigación. Sin embargo, no siempre existe el necesario consenso sobre cómo realizar el acercamiento metodológico a la realidad (Sánchez-Gómez, 2015). 
Aunque es difícil indicar de forma precisa el inicio de los diferentes enfoques investigadores, puesto que el debate sobre este asunto es tan antiguo como la propia historia del pensamiento, encontramos en la antigua Grecia, con Platón, los orígenes pre-cuantitativos en su afán por la abstracción, el idealismo y las matemáticas, o un carácter cualitativo defendiendo el trato directo de las experiencias, abanderado por Aristóteles (Conde,1995). Este enfrentamiento metodológico se dará a lo largo del desarrollo de la ciencia, con unos inicios de una progresiva matematización del mundo, donde todo lo no cuantificable va a ser considerado ruido informativo. La metodología cuantitativa, con procedencia de distintas fuentes, dominará el panorama investigador durante siglos desarrollando una evolución continua y transfiriendo los métodos de las ciencias naturales y experimentales al terreno de la investigación social, en cuyo ámbito se consideran los orígenes la obra de Augusto Comte (1798-1857) y Emile Durkheim (1858-1917) con la influencia significativa de Francis Bacon, John Locke y Emmanuel Kant (Hernández-Sampieri, Fernández-Collado y Baptista, 2010).

Si bien hay consenso en señalar que la observación descriptiva, las entrevistas y otros métodos cualitativos son tan antiguos como la historia escrita (Taylor y Bodgan, 1987: 17), lo cierto es que lo que ahora se denominan métodos cualitativos fueron empleados conscientemente en la investigación social solo a partir de finales del siglo XIX. Todos los autores coinciden en señalar que el análisis de la evolución histórica de la investigación cualitativa no puede desligarse del estudio de otras Ciencias Sociales con las que tuvo una estrecha relación en sus orígenes, fundamentalmente la Sociología y la Antropología. En los años de 1920 se encuentran los momentos clave de dicho enfoque, con el surgimiento de un grupo de investigadores conocidos como la Escuela de Chicago, quienes realizan diversos estudios cualitativos.

El enfoque cualitativo presentará altibajos derivados del posicionamiento del, considerado como su opositor, enfoque cuantitativo. Siguiendo a Hernández Pina (2001, p. 43), podemos decir, que en la década de los sesenta se produce un nuevo resurgir de la metodología cualitativa al incorporarla los profesionales de la educación a sus investigaciones. Hasta esas fechas, los estudios cualitativos de aspectos educativos habían sido desarrollados por antropólogos o sociólogos; y desde entonces, los profesionales de la educación manifestaron progresivamente su interés por la utilización de los métodos y estrategias cualitativas.

Mertens, Bazeley, Bowleg, et al. (2016) recogen las reflexiones realizadas por los expertos y ponen de manifiesto que la investigación con métodos mixtos (MM) se ha ampliado en las últimas décadas con abundantes publicaciones. La aparición del Journal of Mixed Methods Research (JMMR), y el establecimiento de la Asociación Internacional de Investigación de Métodos Mixtos (MMIRA) hacen vislumbrar un desarrollo prometedor para los MM entre los años 2016 y 2020. Mertens et al. (2016) recuerdan que cuando desarrollamos experiencia en el uso de métodos donde nos sentimos cómodos, es difícil romper con esta inercia. Pero extendiendo y afinando las habilidades metodológicas, podemos aumentar el pensamiento conceptual, ver nuevas formas de responder a las preguntas de investigación, e incluso identificar preguntas que no se nos habrían ocurrido de otro modo (Edwards, 2008). En este aspecto los MM pueden desempeñar un papel clave, porque al combinar e integrar métodos cuantitativos y cualitativos, el investigador está motivado para desarrollar un conjunto más amplio de habilidades 
de investigación. Tashakkori y Teddlie (2003) indicaron que los estudios mixtos pueden ser más enriquecedores que los otros enfoques, ya que pueden responder a preguntas de investigación que las otras metodologías no pueden. Como conclusión a las reflexiones de la MMIRA en este tema, Mertens et al. (2016) señalan que algunas preguntas no necesariamente pueden ser categorizadas como cuantitativas, cualitativas o MM. Obviamente, cualquier pregunta de la investigación debe ser potencialmente investigable, pero la clave es descubrir cómo. Puede ser atrevido escribir preguntas al principio que "dicten" los métodos que van a ser usados para responderlas, ya que esto podría engendrar una "visión de túnel" conceptual, evitando que el investigador vea enfoques y datos alternativos que puedan contribuir a responder la pregunta. Por lo tanto, los autores argumentan que las preguntas no asumen necesariamente métodos, aunque puede ser que algunos métodos sean más apropiados que otros para responder a ciertos tipos de preguntas. Además, a medida que se avanza en la investigación, las preguntas se pueden modificar en respuesta a los análisis en curso.

La divulgación de resultados científicos, obtenidos con cualquier tipo de enfoque metodológico pero con unos criterios de calidad, es necesaria para el avance de la ciencia. Hoy día, gracias a los avances tecnológicos, la adquisición del conocimiento es más accesible. Las diferentes bases de datos digitales proporcionan un gran apoyo a la investigación, además de suponer un reconocimiento a los esfuerzos realizados por la comunidad científica y académica.

\section{Método}

\subsection{Objetivos y preguntas de investigación.}

Teniendo en cuenta la evolución de los enfoques metodológicos en las ciencias, se realiza un análisis bibliométrico descriptivo de la investigación en el ámbito de las ciencias sociales, tratando de dar respuesta a cuál es la tendencia en los últimos años de la metodología empleada, qué enfoques dan respuesta de forma más habitual a diferentes tópicos y si hay una tendencia marcada por aspectos geográficos o idiomáticos.

\subsection{Procedimiento}

El estudio parte de la búsqueda realizada en dos bases de datos internacionales, la web of science y scopus, accediendo a ella a través de la licencia de una universidad pública, la universidad de Salamanca. Ambas bases proporcionan referencias bibliográficas y citas de publicaciones periódicas que cumplen con las normas de calidad científica.

La web of science (WoS) es una plataforma Web, propiedad de Clarivate Analytics, que integra otras bases de datos como son Colección principal de Web of Science, Current Contents Connect, Derwent Innovations Index, Korean Journal Database, Medline, Russian Science Citation Index, SciELO Citation Index, abarcando cualquier disciplina del conocimiento, tanto científico como tecnológico, humanístico y sociológicos en algunos casos desde 1900 hasta la actualidad. Su consulta permite el conocimiento de las principales referencias académicas, a la vez que proporciona herramientas de evaluación de la calidad científica. Por su parte Scopus, propiedad de Elsevier, recoge resúmenes y referencias de documentos académicos sobre ciencias, medicina, 
tecnología y ciencias sociales desde 1996, realizando actualizaciones semanales, además de disponer de dos métricas de factor de impacto de la investigación como son Scimago Journal Rank (SCR) y SNIP (Source-normalized impact Paper) de la Universidad de Leyden (FECYT, 2018).

La búsqueda se realizó utilizando los descriptores de "qualitative", "quantitative", "mixed", añadiendo "research" y " study" para hacer distinción en cada uno de los métodos y, en cada una de las bases, refinando posteriormente las búsquedas al área de Ciencias Sociales. Se ha utilizado como rango temporal el periodo de los últimos veinticinco años para obtener los indicadores de cada tipo de estudio, abarcando el periodo de 1993, momento en el que además se dispone de software para el análisis de investigación cualitativa, hasta el 31 de diciembre de 2017.

El procesamiento digital de la información se realizó con un el paquete Microsoft Excel, permitiendo la comparación de datos de las diferentes búsquedas. Finalmente se realiza el análisis de la productividad anual, temática, documental, geográfica e idiomática.

\section{Resultados}

La producción científica registrada en WoS en los últimos veinticinco años ha sido de un total de 1.920.181 documentos de los cuales 901.811 se clasifican en métodos míxtos, 688.976 presentan una investigación cuantitativa y 329.394 se indica el uso de un método cualitativo. Por su parte Scopus facilita 1.391.592 archivos de los cuales 662.530 son de carácter cuantitativo, 390.483 son estudios mixtos y 338.579 presentan una investigación cualitativa.

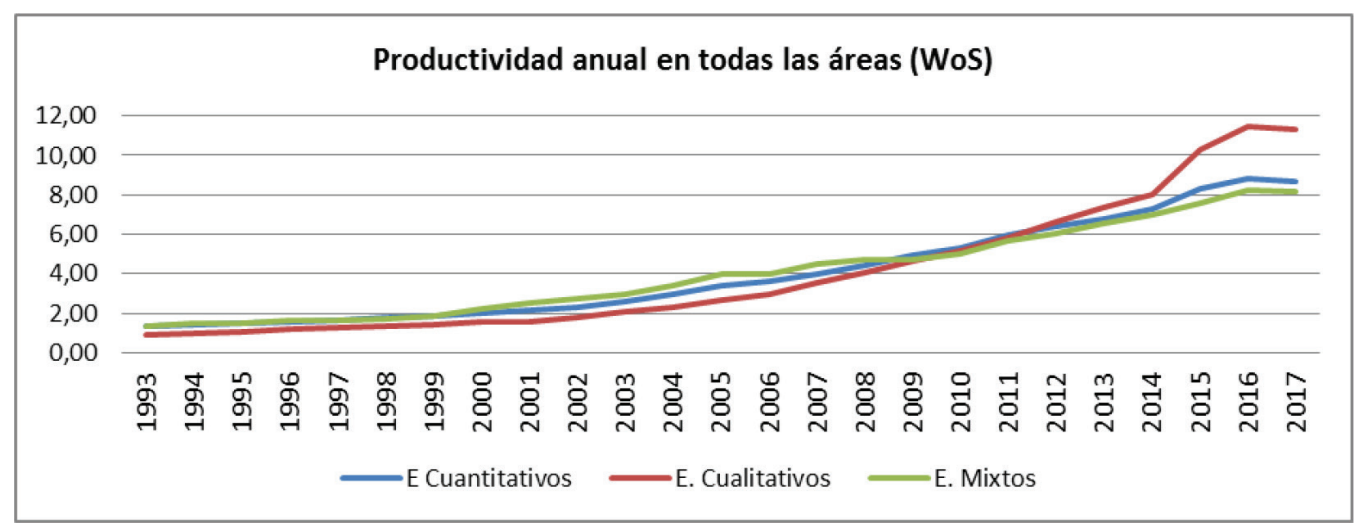

Figura 1 - Productividad anual de acuerdo a Web of Science.

En la figura 1 se presentan los resultados en porcentajes de los últimos veinticinco años de productividad científica registrados en la bases de datos Web of Science, al igual que en la base de datos de Scopus (fig. 2).

La WoS ofrece información en relación a las categorías generales, pudiendo observar en la figura 3 como las ciencias de la tecnología, durante el periodo de tiempo seleccionado, 
es el ámbito que más producción científica desarrolla, encontrándose las ciencias sociales en el quinto puesto.

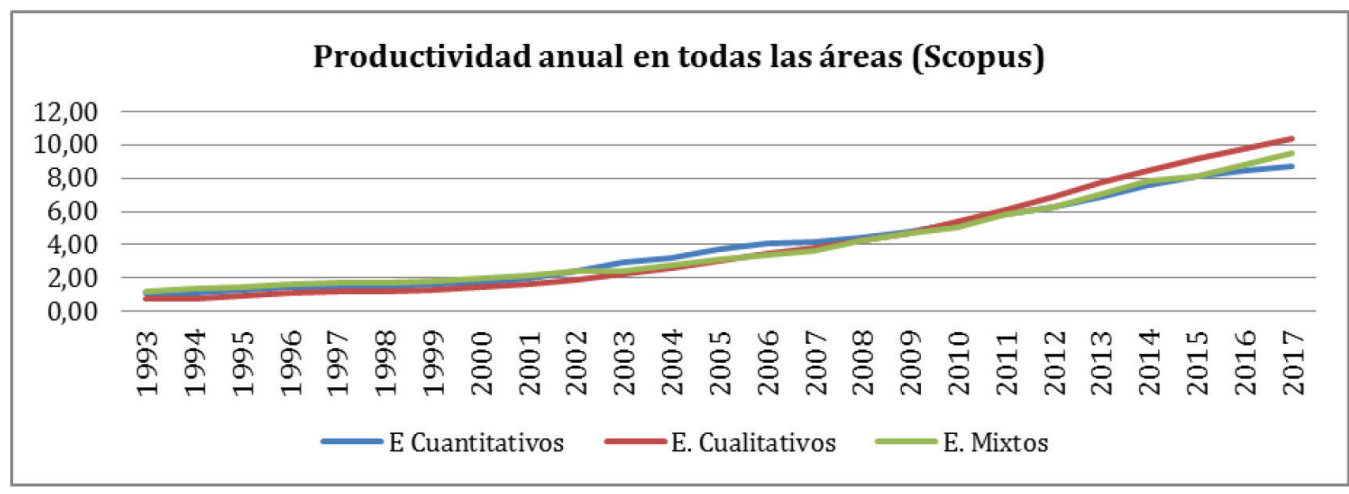

Figura 2 - Productividad anual de acuerdo a Scopus.

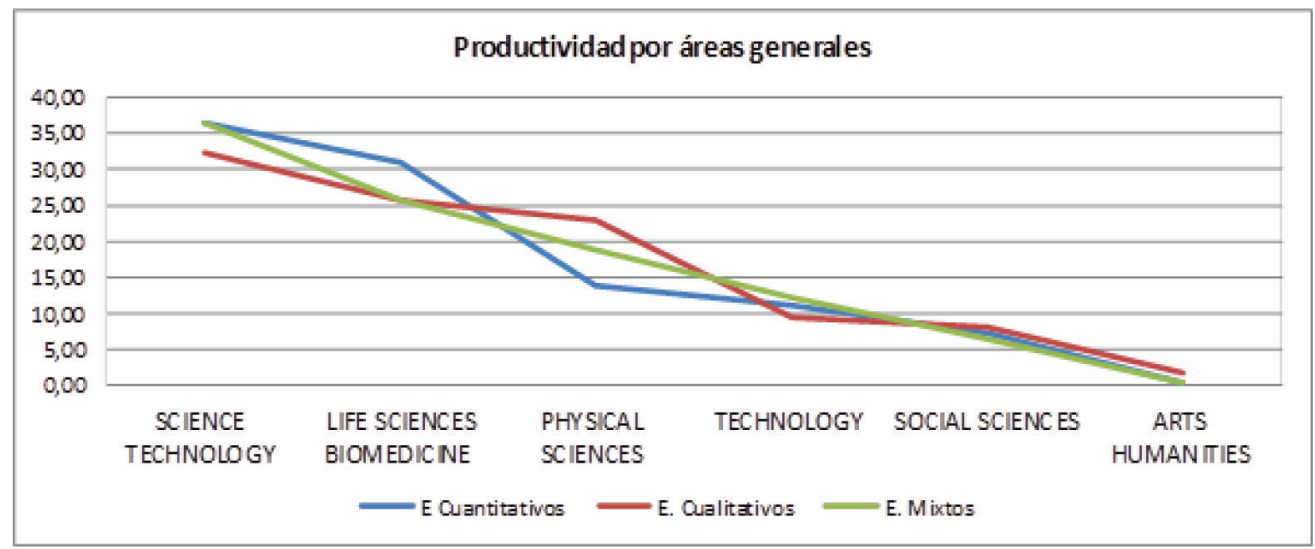

Figura 3 - Productividad anual por áreas generales (WoS).

Para llevar a cabo el análisis de la productividad de acuerdo al país, se han elegido los cinco primeros países que ofrece cada base de datos en cada uno de los métodos de investigación utilizado. Al no coincidir el orden del país en todos los tipos de investigación, se han elegido los cinco primeros de cada tipo de investigación y se han incluido los datos del país, que aun no siendo el quinto en ese método, si se ofrecen los datos, los resultados se ofrecen en la figura 4.

Analizando de forma específica el área de las ciencias sociales, se encuentran registrados 462.284 materiales de carácter científico durante el periodo de 1993 hasta 2017, de los cuales 128.161 recogen estudios de carácter cuantitativo, 150.765 abordan métodos mixtos y 183.358 son documentos de investigación cualitativa. En la figura 5 se muestra la evolución correspondiente a la base de datos Wos. Se observa como en WoS la productividad científica en el área de las ciencias sociales, en especial, ha experimentado 


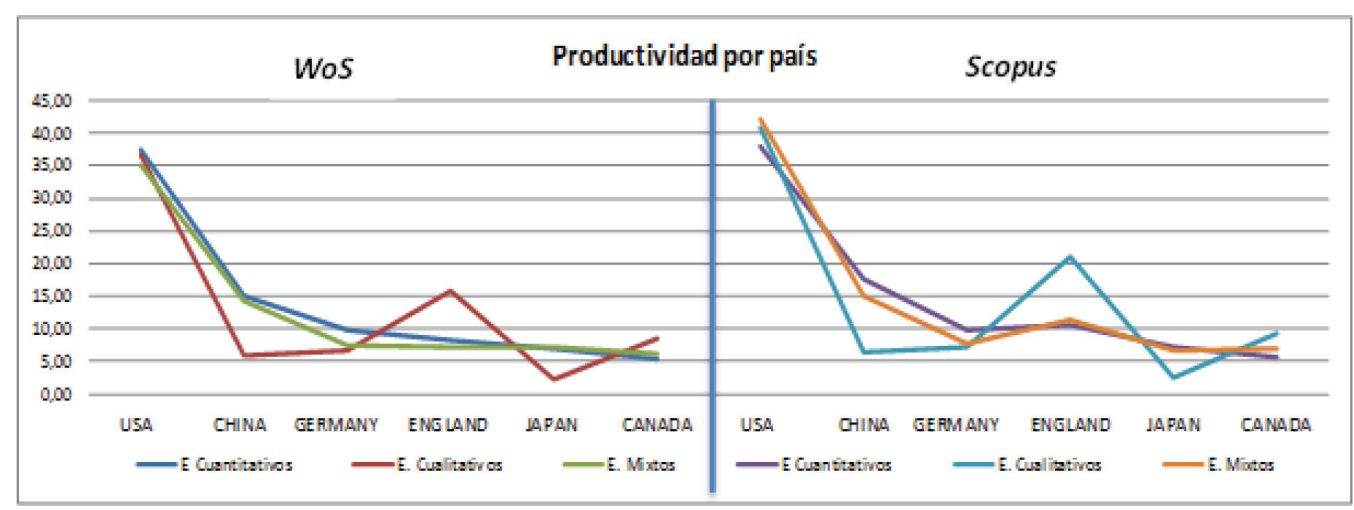

Figura 4 - Productividad por países áreas generales. Comparación de bases.

un mayor crecimiento que en la producción científica en general, y aunque los inicios de dicha evolución han sido más modestos, en los últimos años ha superado a la productividad general, destacando en el año 2016 en el cual hay una diferencia de 3,83 puntos en los enfoques mixtos a favor de las ciencias sociales frente a las ciencias en general. Al igual que el último año 2017 se produce un descenso de la producción en la literatura científica en general, este se ha acentuado en el ámbito de las ciencias sociales siendo más evidente en los métodos cualitativos.

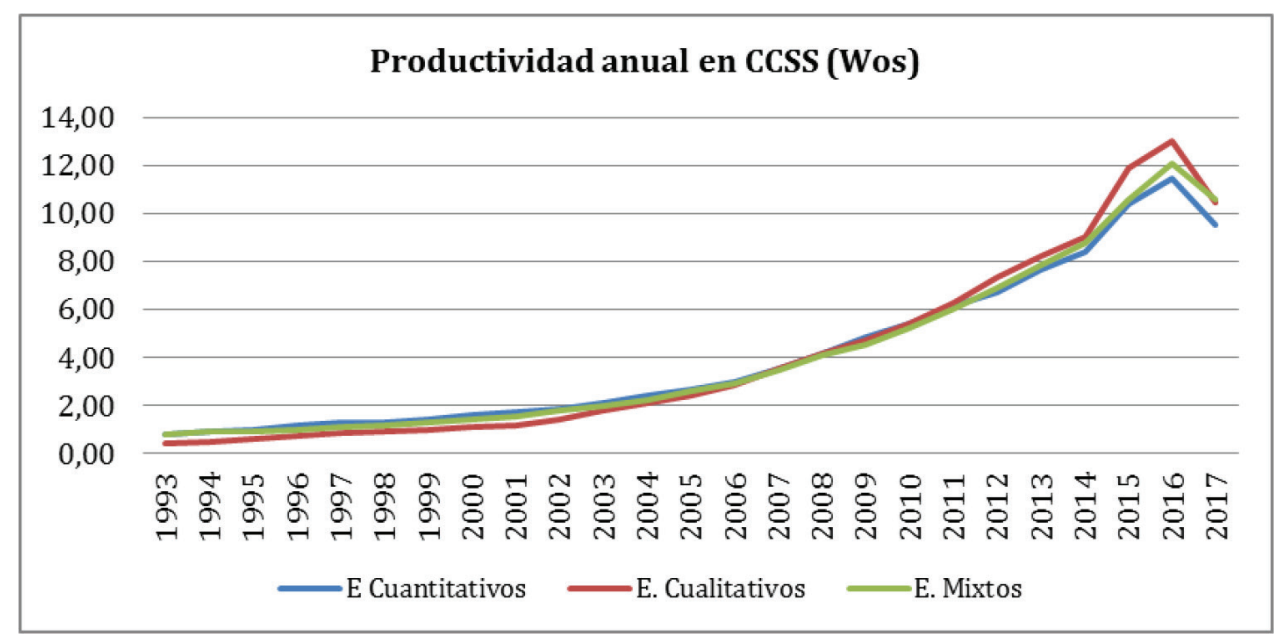

Figura. 5 - Productividad anual en el área de las Ciencias Sociales. Base de datos Web of Science.

En la base de datos Scopus, se observa la misma evolución que la manifestada en la base WoS, con datos muy similares en el año 2016. La evolución progresiva de la productividad científica en las ciencias sociales, como se refleja en la figura 6 ha sido superior a la literatura científica general, siendo más evidente en los métodos mixtos, con una diferencia también de 3,84 puntos, de acuerdo a los datos ofrecidos por esta 
base de datos, quedando los métodos cualitativos una distancia de 1, 49 puntos frente a 2,68 de los métodos cuantitativos.

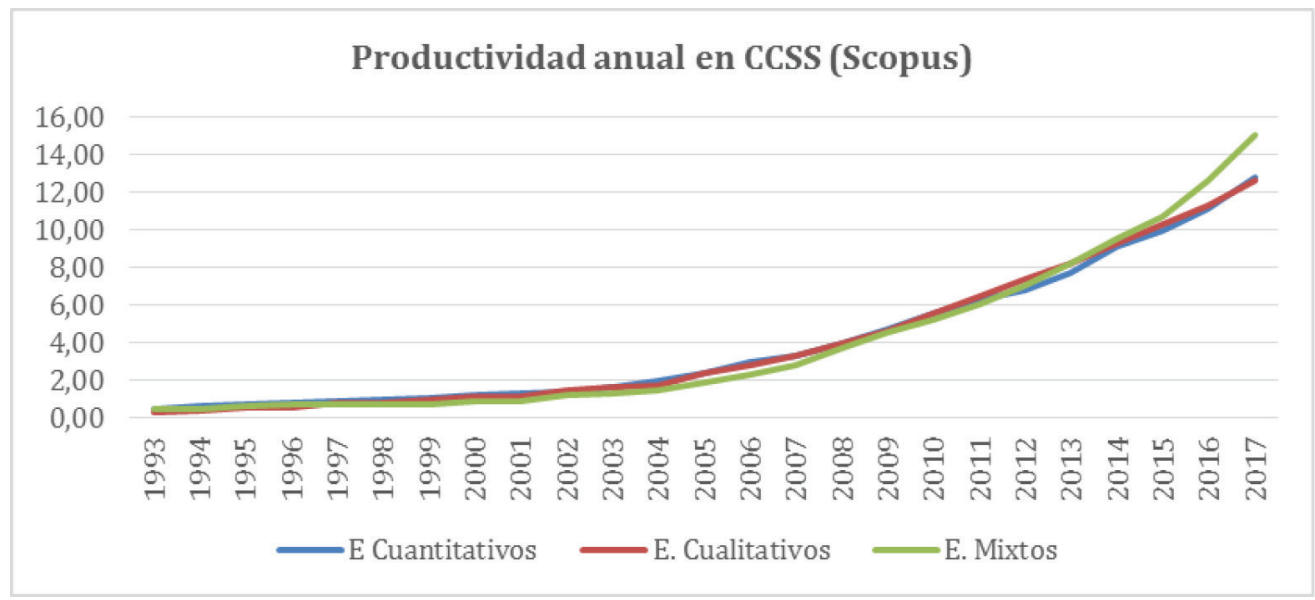

Figura. 6 - Productividad anual área de las Ciencias Sociales. Base Web of Science.

Con el fin de analizar la productividad por países en el área de las ciencias sociales, se ha seguido el mismo criterio de elegir los cinco primeros países de cada método y para cada base, añadiendo, por motivos de interés de los autores, los países de España (que en los métodos cuantitativos según la Wos se encontraría en el séptimo lugar), Brasil y Portugal. Como se presenta en la figura 7 el país con mayor productividad en esta área sigue siendo Estados Unidos, como ocurre con la literatura general, seguido del Reino Unido, que se encuentra en el cuarto lugar cuando se analizan todas las áreas. En el tercer puesto encontramos en la base de datos WoS a la República popular China, que por motivos de ausencia de datos en Scopus no se ve reflejada.

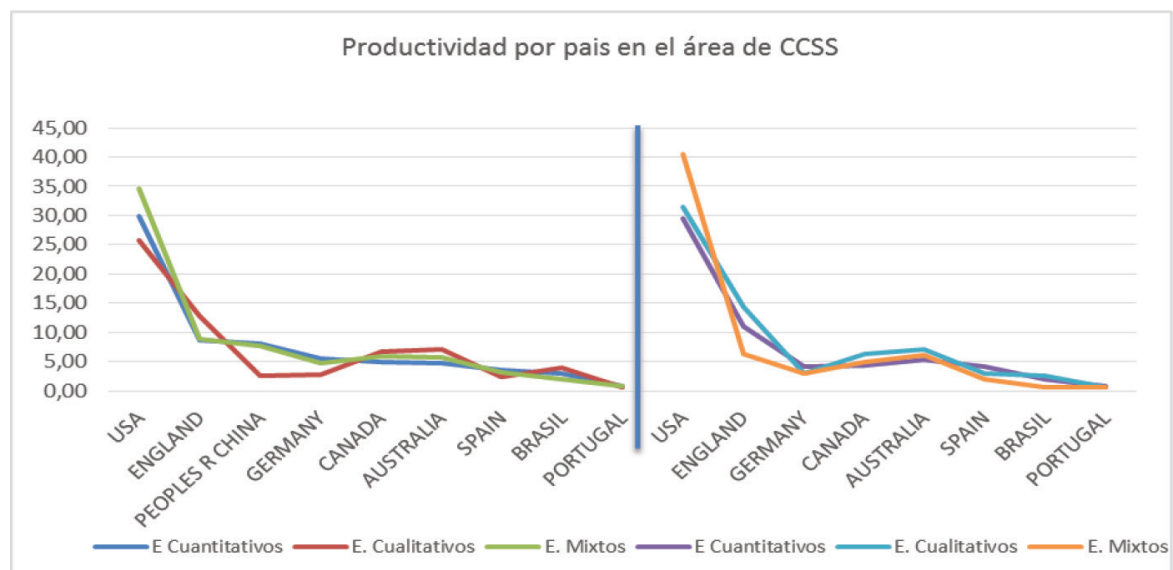

Figura. 7 - Productividad anual área de las Ciencias Sociales. Base Web of Science. 
En relación al idioma vehicular de la productividad, como era de esperar, la gran mayoría se realiza en inglés, como puede observarse en la figura 8, seguido del koreano, español, portugues, ruso y francés en prácticamente los mismos porcentajes, que aunque ínfimos se encuentran representados en los primeros puestos. Como se observa no hay diferencias apreciables de acuerdo al tipo de metodología empleada.

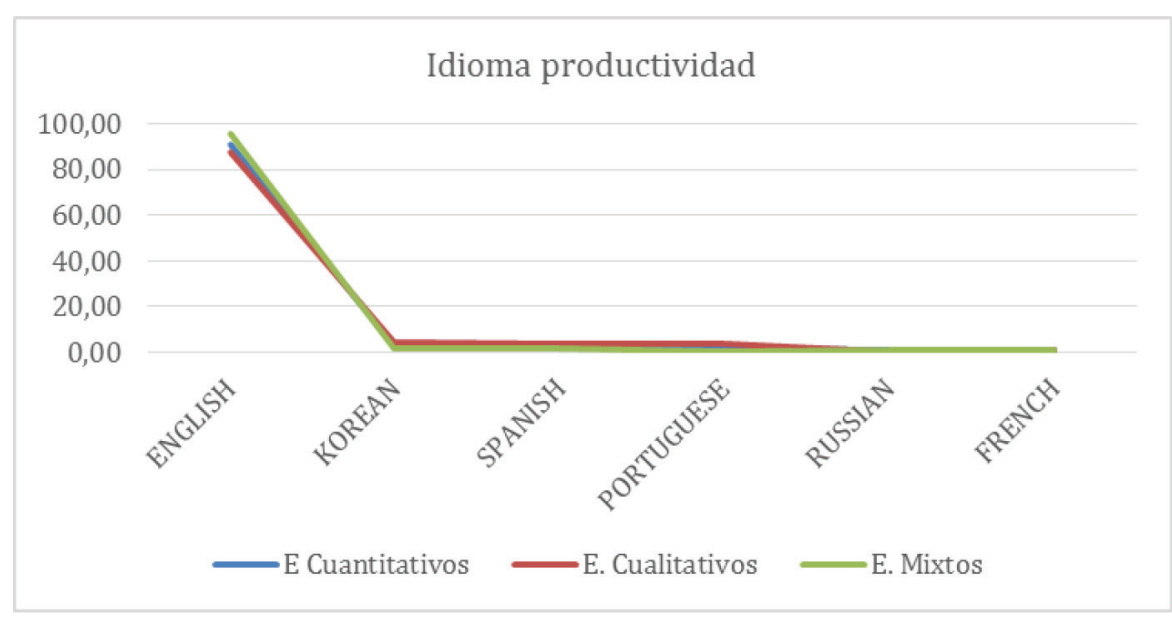

Figura. 8 - Idioma de la literatura científica en el área de las Ciencias Sociales (Web of Science).

En relación al tipo de documentos a los que se puede acceder, tanto a nivel general como en el área de las ciencias sociales, una amplísima mayoría son artículos de revistas.

\section{Conclusiones}

Los resultados manifiestan la gran literatura científica de la que puede disponer un investigador tanto como base para el inicio de sus investigaciones, como para avalar, o refutar, los resultados obtenidos, permitiendo a su vez el crecimiento del conocimiento. Como se observa, los resultados ofrecidos por las dos bases de datos son complementarios. Se recomienda el uso de ambas bases, para abarcar el objetivo de estudio, puesto que según el área de investigación, Scopus puede ser menos exhaustiva en referencias anteriores a 1996, y sobre todo cuando se desee analizar la calidad de revistas puesto que presentan comportamientos diferentes en algunos indicadores (Hernández-González, Sans-Rosell, Deltell y Reverter, 2016).

En relación a la evolución anual se observa un gran crecimiento de la productividad científica, siendo esta más lenta en el área de las ciencias sociales en un inicio y ascendiendo con los inicios del siglo XXI. Aunque se observa un ligero descenso en el último año.

En cuanto a las áreas de mayor productividad científica se encuentran las ciencias tecnológicas, biomedicina y física, estando en una quinta posición las ciencias sociales seguida de las humanidades. Sin embargo, teniendo en cuenta el ascenso superior en 
productividad archivística en relación a la literatura en general, se dirige a obtener un mejor posicionamiento.

El país que mayor productividad genera es Estados Unidos, tanto a nivel general como en Ciencias Sociales, sin embargo dependiendo del área se observa un cambio geográfico en la productividad. Así mientas China se posiciona en segundo lugar en literatura científica en general, en el área de las Ciencias Sociales es el Reino Unido quien ostenta el segundo lugar.

En relación al idioma, y como era de esperar, el inglés es el lenguaje principal, debido a que es requisito fundamental para la publicación de un artículo en muchas revistas, convirtiéndose así en el idioma vehicular de la ciencia. Sin embargo, no se debe desdeñar el uso del español y portugués como lenguas científicas minoritarias, teniendo en cuenta que también reciben citaciones bibliográficas aquellas revistas que permiten la presentación de artículos en dicho idioma (Quindón, 2009). Como manifiesta Aréchaga (2011) los propios científicos de un país son los que deben ayudar a potenciar el idioma oficial de su país, no menospreciando las revistas nacionales en pro de las americanas, a las que se considera valedoras de sus mejores trabajos.

Los resultados de los análisis bibliométricos efectuados en este estudio demuestran que en los últimos años los avances de los estudios cualitativos y mixtos han sido espectaculares, a pesar de que son escasas las propuestas sobre los procedimientos metodológicos que integren realmente estrategias cualitativas y cuantitativas (Flick (2014). Los métodos mixtos se han impuesto como un enfoque de investigación autosuficiente, gracias a autores como Tashakkori y Teddlie (2003, 2009); Johnson, Onwuegbuzie y Turner (2007); Bazeley (2009); Creswell (2011); Fakis, Hilliam, Stoneley y Townend (2014), Creswell y Plano Clark (2011), Natasi, Hitchcook y Brown (2010), Fetters, Curry y Creswell (2013), Morse y Niehaus (2009) y Anguera (2018), que han creado e impulsado las potencialidades, características y nuevas técnicas metódicas en la abundante producción científica que han elaborado.

Recuérdese que con la perspectiva cualitativa se concibe la realidad como una construcción del ser humano, es decir, el sujeto parte de una serie de esquemas mentales que ya posee, y gracias a la interacción con otras experiencias, construye nuevos conocimientos. El aprendizaje, por tanto, requiere de la participación activa y subjetiva del aprendiz (Bedoya y Arango, 2013). Si, además, incorporamos elementos de carácter cuantitativo, nos interesamos no solo por el proceso, sino también por el producto o resultado. Utilizar ambos enfoques robustece los procedimientos y proporcionan más calidad a la investigación con acciones de triangulación. Delgado (2014) refuerza esta idea señalando que utilizando los dos en un mismo estudio, se combina el rigor formal de la cuantitativa y la creatividad y plasticidad de la cualitativa; y que este maridaje no conlleva una mera yuxtaposición, sino combinación flexible de etapas de la investigación y de sus componentes

De los párrafos anteriores se desprende que no todas las observaciones son idóneas de medición cuantitativa, más aún cuando se trabaja sobre cuestiones de las particularidades e intereses de los participantes y la captación de sus relatos. La comparación exige establecer diferenciaciones no sólo en función de la cantidad (cuantitativas), sino también de la cualidad (cualitativas). Por lo tanto, el rasgo principal que caracterizan los métodos mixtos es la coexistencia de elementos cualitativos y cuantitativos en los procesos de 
investigación. En estos métodos se recogen los datos mediante diversidad técnica, instrumentos y herramientas de recogida de información, en su mayoría cualitativas (observación, entrevistas en profundidad, relatos, historias de vida, autobiografías), y otras de carácter cuantitativo (ejemplo, cuestionarios).

Acorde con las conclusiones del estudio bibliométrico que se presenta en este documento, Anguera \& Hernández (2016), avalan nuestros resultados confirmando en su artículo sobre los métodos mixtos, la existencia de un número cada vez mayor de artículos sobre investigación cualitativa y sobre todo métodos mixtos, en las revistas científicas actuales y especializadas, hecho que se pone de manifiesto en congresos internacionales que se suceden en diversos lugares del mundo. El SXXI ha sido altamentefructífero en las ciencias sociales y de la salud en el desarrollo y evolución de enfoques alternativos al positivismo clásico, como se demuestra en este trabajo En la actualidad, podemos afirmar de forma categórica que la culminación de esfuerzos de actividades académicas, de investigación, documentos científicos y los encuentros científicos como los CIAIQ (2013- 2014-20152016-2017, 2018) han tenido su fruto en la comunidad científica (Onwuegbuzie \& Poth, 2015; Costa, Sánchez Gómez y Martín Cilleros, 2017) y en la denominada "nueva era” de los enfoques de investigación (Tashakkori \& Cresswell, 2007). Este proceso, hemos de decirlo, no ha estado exento de polémicas (Creswell, 2011).

Anguera et al. (2016) afirma, que pocas temáticas que existen en metodología de investigación han producido tanta literatura como la que se ha generado, en las dos primeras décadas del siglo XXI, con métodos mixtos. Sostiene que son muchos los autores e investigadores que quieren complementar o integrar ambas metodologías científicas en sus trabajos de investigación: proyectos, tesis, trabajos fin de máster, etcétera.

Las conclusiones del estudio presentado ofrecen un futuro esperanzador para el entendimiento entre las dos metodologías. Tras un largo y duro periodo de tiempo en el que se ha mantenido el enfrentamiento o la imposibilidad de comunicación entre ambas, a finales del siglo XX se empezó a vislumbrar la posibilidad de complementación entre los enfoques cualitativo y cuantitativo dirigida por los métodos mixtos. Fetters \& Freshwater , citado por Anguera et al. (2016), afirma que el reto actual no solo es la complementariedad sino la integración de las dos metodologías. Esta integración consiste en "la producción de algo nuevo que tiene más valor que la suma de las partes. Y lo que cuantitativamente se indica, en el título, que $1+1=3$, significa que cualitativo+ cuantitativo es más que la suma de ambos" (p. 18).

Desde esta perspectiva, y siguiendo los planteamientos de Anguera et al. (2016), se aconseja a todos los investigadores que se preocupen por gestionar los resultados de los dos enfoques de forma integrada y no cada uno por separado. En la actualidad se dan las condiciones oportunas y favorables para que esto sea así. Los estudios metodológicos de integración permiten avanzar en el perfeccionamiento de los diseños y procedimientos metódicos de este nuevo paradigma integrador denominado Mixed Methods, métodos mixtos.

\section{Referencias}

Anguera Argilaga, M. T., \& Hernández Mendo, A. (2016). Avances en estudios observacionales de Ciencias del Deporte desde los mixed methods. Cuadernos de Psicología del Deporte, 16 (1), 17-30. 
Aréchaga, J. (2011). El prestigio y la rentabilidad de las revistas científicas españolas se basa en el uso internacional de sus contenidos. Endocrinol. Nutr., 58(2), 57-61.

Bazeley, P. (2009). Editorial: Integrating data analyses in mixed method research. Journal of Mixed Methods Research, 3(3), 203-207.

Bedoya, M. E. A., \& Arango, P. E. (2013). Constructivismo y construccionismo social: Algunos puntos comunes y algunas divergencias de estas corrientes teóricas. Revista de Trabajo Social e Intervención Social, 17, 353-378.

Conde, F (1995). Las perspectivas metodológicas cualitativa y cuantitativa en el contexto de la historia de las ciencias. En: Delgado, J. M. \& Gutiérrez, J. (Comps.). Métodos y técnicas cualitativas de investigación en ciencias sociales. Madrid: Editorial Síntesis S.A.

Costa, A.P., Sánchez-Gómez, M.C., \& Martín-Cilleros, M.V. (2017). La práctica de la investigación cualitativa: ejemplificación de estudios. Oporto: Ludomedia.

Creswell, J.W. (2011). Controversies in mixed methods research. En: N.K. Denzin \& Y.S. Lincoln (Eds.), The Sage handbook of qualitative research (4th ed.) Thousand Oaks, CA: Sage.

Creswell, J. W., \& Plano Clark, V. L. (2011). Designing and conducting mixed methods research ( $2^{\text {nd }}$ ed.). Thousand Oaks, CA: Sage.

Edwards, J.R. (2008). To prosper, organizational psychology should ... overcome methodological barriers to progress. Journal of Organizational Behavior, 29, 469-491

Fakis, A., Hilliam, R., Stoneley, H., \& Townend, M. (2014). Quantitative analysis of qualitative information from interviews: A systematic literature review. Journal of Mixed Methods Research, 8(2), 139-161.

Fetters, M. D., Curry, L. A., \& Creswell, J. W. (2013). Achieving integration in mixed methods designs: principles and practices. Health Services Research, 48(6 Pt. 2), 2134-2156.

Fundación Española para la Ciencia y la Tecnología (2018). Web of Science. Recuperado de https://www.fecyt.es/es/recurso/web-science.

Fundación Española para la Ciencia y la Tecnología (2018). Scopus. Recuperado de https://www.fecyt.es/es/recurso/scopus.

Hernández-González, V., Sans-Rosell, N., Jové-Deltell, M. C, \& Reverter-Masia, J. (2016). Comparación entre Web of Science y Scopus, Estudio Bibliométrico de las Revistas de Anatomía y Morfología. International Journal of Morphology, 34(4), 1369-1377. https://dx.doi.org/10.4067/So717-95022016000400032

Hernández Sampieri, R., Fernández, C., \& Baptista, P. (2010). Metodología de la Investigación. México D.F.: McGraw-Hill

Hernández Pina, F. (2001). Bases metodológicas de la investigación educativa. Barcelona. PPU. 
Johnson, R. B., Onwuegbuzie, A. J., \& Turner, L. A. (2007). Toward a definition of mixed methods research. Journal of Mixed Methods Research, 1(2), 112-133.

Quindós, G. (2009) Confundiendo al confuso: reflexiones sobre el factor de impacto, el índice h(irsch), el valor $\mathrm{Q}$ y otros cofactores que influyen en la felicidad del investigado. Rev. Iberoam. Micol., 26(2),97-102.

Natasi, B., Hitchcock, J., \& Brown, L. (2010). An inclusive framework for conceptualizing mixed methods design typologies: Moving toward fully integrated synergistic research models. En: A. Tashakkori \& C. Teddlie (Eds.) Handbook of mixed methods in social \& behavioral research. Thousand Oaks, CA: Sage

Mertens, D. M., Bazeley, P., Bowleg, L., Fielding, N., Maxwell, J., Molina-Azorin, J. F., \& Niglas, K. (2016). The future of mixed methods: A five year projection to 2020. Mixed methods international research association. Journal of Mixed Methods Research, 11(1) 11-18.

Morse, J., \& Niehaus, L. (2009). Mixed method design: Principles and procedures. Walnut Creek, CA: Left Coast Press.

Onwuegbuzie, A. \& Poth, C. (2015). Editor's afterword, Special issue: Mixed Methods. International Journal of Qualitative Methods, 14(2), 122-125.

Tashakkori, A. \& Cresswell, J.W. (2007). The new era of mixed methods. Journal of Mixed Methods Research, 1(1), 3-7.

Tashakkori, A., \& Teddlie, C. (2003). The past and future of mixed methods research: From data triangulation to mixed model designs. En: A. Tashakkori \& C. Teddlie (Eds.), Handbook on mixed methods in the behavioral and social sciences. Thousand Oaks, CA: SAGE.

Taylor, S. J., \& Bogdan, R. (1987). Introducción a los métodos cualitativos de investigación (Vol. 1). Barcelona: Paidós.

Sánchez-Gómez, M.C. (2015). Metodología de investigación en pedagogía social, avance cualitativo y modelos mixtos. Pedagogía Social. Revista Interuniversitaria, 25, 21-34. 